\title{
¿Hacia unas Nuevas Matemáticas?
}

\author{
Chamoso Sánchez, J. Ma \\ Facultad de Educación. \\ Univ. Salamanca (España) \\ Rawson, W.B. \\ School of Education. \\ Univ. Exeter (England)
}

\begin{abstract}
"Desarrollar ciertas actitudes forma parte, a veces implícitamente, de los objetivos de la enseñanza: es, en todo caso, el fin de la educación. Algunos piensan, incluso, que es más importante desarrollar actitudes que conocimientos".

J.P. Guichard
\end{abstract}

En los últimos resultados dados a conocer en 1997 por el TIMSS (Tercer Estudio Internacional de Matemáticas y Ciencias), después de un estudio realizado en más de 45 países en el curso 94/95, se considera que "en todos los países del mundo las matemáticas son un papel importante del currículum escolar, y se consideran materias esenciales para la formación de los jóvenes (...) Es considerado como un medio para desarrollar en el individuo hábitos de razonamiento riguroso y crítico". Los resultados del rendimiento español en los cursos de $7^{\circ}$ y $8^{\circ}$ de E.G.B. (en los que se efectuaron las pruebas) son, en ambos casos, inferiores al rendimiento medio internacional.

Observamos los Datos Básicos sobre la Evaluación de la Educación Primaria en España (Instituto Nacional de Calidad y Evaluación, I.N.C.E., 1995) referidos al rendimiento académico de los alumnos de 12 años $\left(6^{\circ}\right.$ Curso). Se puede ver que, después de aplicar las diferentes pruebas y cuestionarios, es en el área de Matemáticas donde el porcentaje de respuestas correctas es menor (aproximadamente el $50 \%$ ) si lo comparamos con las demás áreas de estudio (Lengua Castellana y Literatura, 64 \%; Ciencias Sociales, $61 \%$, y Ciencias de la Naturaleza, $64 \%)$.

Una razón que induce al estudio del proceso de enseñanza-aprendizaje de las Matemáticas deriva de la importancia social que se le da a esta asignatura. Es quizás la materia más prestigiada socialmente y a la que se le atribuye cierto valor predictivo sobre las capacidades del propio individuo. "La realidad es que permanece muy extendido el 'mito de las Matemáticas', según el cual los niveles de inteligencia, el triunfo social e incluso las expectativas del futuro bienestar están en relación directa con las buenas calificaciones en esta área" (Guerrero Ojeda, 1989: p. 57). Malén Aznárez (1997: p. 78) califica las Matemáticas como "la materia que ha sido para generaciones de españoles, y aún lo es para muchos, el coco y pesadilla de sus años de estudiante. Una pesadilla irremediable porque los niños aprenden desde bien pequeños que la primera nota por la que se interesan sus padres es por la de matemáticas".

Siempre se ha escuchado que es la disciplina que resulta más difícil a los estudiantes. Hoy siguen teniendo validez las palabras de Dienes, escritas en 1964 (citado por Alcalá): "Actualmente son muy pocos los profesores de matemáticas, cualquiera que sea el nivel en que trabajan, que se encuentren satisfechos del modo en que transcurre su enseñanza. Efectivamente, son muchos los niños que sienten antipatía por las matemáticas -antipatía que aumenta con la edad- y muchos los que encuentran dificultades casi insuperables en las cuestiones más sencillas. Hay que reconocer que la mayor parte de los niños nunca llega a comprender la significación real de los conceptos matemáticos. En el mejor de los casos, se convierten en consumados técnicos en el arte de manejar complicados conjuntos de símbolos, pero la mayor parte de las veces acaban de desistir de comprender las imposibles situaciones en que las exigencias de las matemáticas escolares de hoy les colocan. La actitud más corriente consiste, simplemente, en esforzarse en aprobar un examen, tras lo cual nadie dedica a las matemáticas ni un pensamiento más. Con muy pocas excepciones, esta situación se puede considerar lo bastante general como para llamarla normal".

Cuenta el profesor Cuesta Dutari, en el prólogo de su libro La Sinfonía del Infinito (1981: p. 4), que atribuyen a D. Miguel de Unamuno esta agudeza, dicha sin duda pensando en alguien: "quería saberlo todo; ;figúrense lo tonto que sería!" Y Constantino de la Fuente y el grupo DECA (1990: p. 144-145) dicen que todavía "somos muchos, o al menos demasiados, los profesores y profesoras que queremos enseñarlo todo (...) Hasta el presente, el profesorado ha sido el propagador de conocimientos. El alumnado, con más o menos engaño y de acuerdo con sus capacidades, se ha limitado a recogerlos con el fin de devolvérselos lo más fielmente posible y conseguir la meta del aprobado (...) Con exageración, pero con no poca razón, se ha hablado alguna vez de obtener titulados en serie".

En verdad que es demasiado frecuente en la enseñanza de las Matemáticas la transmisión de conceptos a modo de recta. Parece que se tiene una única idea en la cabeza: aprobar un examen. Esto no ronda únicamente en la mente de muchos alumnos, sino en la de muchos profesores y en la del propio sistema. No debería ser así. El pensamiento matemático no se trasplanta de un individuo a otro, sino que cada uno lo tiene que ir construyendo a partir de su propia experiencia. La enseñanza de las Matemáticas ha de entenderse como un proceso a largo plazo.

\section{Pero, ¿cuáles son las Matemáticas que demanda la sociedad?}

Parece aceptado que las Matemáticas ocupan un puesto especialmente importante entre las materias del sistema de educación de cualquier país. Y hay que reconocer la importancia de su contenido para mantener una parte de la sociedad, capaz de servir a la tecnología, la industria, la ciencia... Únicamente una parte específica va a acceder a esos puestos, pero hay otros muchos ciudadanos que no lo podrán hacer por diversas razones: no hay sitio para todos, no todas las personas tienen esas apetencias y, además, no todos tienen capacidad para ello. Por eso nuestra responsabilidad como profesores es saber diferenciar unas Matemáticas de otras, y considerar cuidadosamente el estilo de enseñanza que adoptamos. Tenemos que reflexionar sobre nuestra propia filosofía de enseñanza-aprendizaje.

Realmente queremos que nuestros alumnos sepan muchas Matemáticas. Pero más que aprender unos cuantos conceptos concretos, nuestra pretensión debe ser una formación integral de la persona. Queremos formar personas que afronten los problemas, superen las dificultades, sepan luchar cuando tengan que hacerlo y renunciar en el momento adecuado. Y tener la humildad de reconocer cuándo se está en el error (no podemos olvidar que muchas veces el error enseña más que el acierto). En definitiva, que sepan ser personas, seres humanos que luchen por conseguir lo que quieren, aunque eso a veces sea duro, y que hagan en cada momento lo que tienen que hacer, tanto en su futura labor 
profesional o docente como en su vida en general. También hay que tener en cuenta las palabras de Al Cuoco (1997: p. 187): "Los estudiantes tienen intereses, vida familiar, cultura y valores diferentes. No importa que estén estudiando, siempre intenté ampliar sus definiciones de la realidad para incluir las matemáticas. Esta tarea es, a veces, difícil pero estoy convencido de que el poder de los que utilizarán las matemáticas en la próxima centuria será, más que ver éstas aumentando con lupa la realidad, verlas como una parte de la realidad".

Recuerdo en una ocasión que hicimos una experiencia de preguntar a varios estudiantes qué detergente les parecía mejor, es decir, cuál era más efectivo, cuidaba más la ropa y lavaba más blanco. Enseguida alguien dijo que Colón y otro le replicó que no, que era mucho mejor Dixan. Otro enseguida dijo que no sabía fijo, pero que él creía que Ariel. Después de una pequeña discusión sobre cuál era el mejor, alguien preguntó: "¿Y cómo podemos saber cuál es el mejor?". Rápidamente contestó el que había dicho Colón: "Yo sé que es Colón porque es el que usa mi madre". Otros corroboraron esa idea, añadiendo que ellos no lavaban nunca y no podían saber cuál era el mejor. Enseguida el último que había hablado dijo que, según eso, tales detergentes no eran los mejores, sino que eran los que usaban sus madres, sin saber siquiera si los usaban porque les parecía mejor, o porque eran más baratos, o quizás lo habían empezado a usar por casualidad y lo seguían haciendo. Es decir, no estaban expresando su opinión, sino la opinión de otras personas, que quizás fuese correcta, o fuese alguien de quien se fiaban mucho, pero eso no significaba que ellos pensasen así. Quizás ellos no pudiesen siquiera responder a esa pregunta, por desconocimiento. Aunque esto no deja de ser más que una anécdota, lo que buscamos es gente crítica, que opine cuando tenga que hacerlo y que calle cuando sea lo adecuado. Y a tal actitud contribuyen mucho las Matemáticas. Hay que tener gran respeto a las ideas y opiniones de los demás, pero no por ello podemos dejar de pensar por nosotros mismos.

A pesar de todo debemos hacer una reflexión. Fijémonos en el sistema de educación de Inglaterra. La enseñanza de las Matemáticas ha estado influida por la introducción del Currículum Nacional en 1989. Unos siete años antes se había publicado un importante informe con una posterior trascendencia muy superior a los límites ingleses, el Informe Cockcroft. Entre las muchas recomendaciones de éste hay una que es la necesidad de relacionar el contenido de los cursos de Matemáticas con las experiencias diarias de los alumnos (DES, 1982, p. 462). Las mismas intenciones aparecen en la sección del Currículum Nacional titulada Utilizar y aplicar las Matemáticas (DfE, 1995, p. 6): "A los alumnos se les deben dar oportunidades para utilizar y aplicar las Matemáticas en tareas prácticas, en problemas de la vida diaria y dentro de las mismas Matemáticas".

Naturalmente, es obvio que el estudio de las Matemáticas debe relacionarse con la vida diaria. No hay nada llamativo en ello. La expresión de las Matemáticas en el Currículum Nacional refleja el punto de vista de los autores, las directrices oficiales de lo que debe ser enseñado. No resulta extraño que haga hincapié en la utilidad, porque es lo que demanda la sociedad. Sin embargo, lo que pide ésta no coincide necesariamente con todo lo que son las Matemáticas. No podemos olvidar la naturaleza de las Matemáticas en sí mismas (que queda reflejado en una parte de la frase anterior, utilizar y aplicar las Matemáticas en las mismas Matemáticas). Quizá sea conveniente considerar diferentes niveles de materia y de enseñanza porque en otro caso consideraríamos la amplia mayoría pero olvidaríamos "los pocos". Éste es un aspecto que no podemos descuidar los que estamos inmersos en la enseñanza-aprendizaje de las Matemáticas.

\section{La importancia de las actitudes}

En las directrices de la Secretaría de Estado de Educación de España donde se establecen orientaciones para la distribución de objetivos, contenidos y criterios de evaluación para cada uno de los ciclos, se hace especial hincapié en la importancia de la actitud positiva de los alumnos hacia cada uno de los diversos contenidos que forman el currículum en estos niveles. También en el boletín de IEPS (Instituto de Estudios Pedagógicos Somosaguas) de diciembre de 1997 se dice que "aprender en la escuela es todavía, en muchas aulas, un puro aprendizaje mental. Las emociones son, más bien, algo a las que no se da ninguna importancia. Pero (...) si el objetivo de la escuela es de verdad preparar para la vida, deberá contribuir al desarrollo de toda la personalidad de los alumnos. Desde hace algunos años, las directrices de la reforma educativa van en esta dirección. El aprendizaje integral no sólo abarca el intelecto, sino que hace referencia también a la emoción, a la intuición y a la acción en el proceso de aprendizaje".

Además, considerando el concepto de actitud, la respuesta parece indudable: la vida del aula conlleva una participación y una serie de intercambios que da pie a un proceso a través del cual, sobre la base de las informaciones recibidas, los sujetos van extrayendo una serie de atributos y formando un conjunto de creencias y actitudes sobre las que diseñan y modelan sus actuaciones con el fin de establecer relaciones satisfactorias con el medio. Por tanto, el estudio de las actitudes puede contribuir a facilitar la comprensión de lo que sucede en las aulas y la dinámica de los centros.

Por otra parte, respecto a la importancia que se da al estudio de las actitudes dentro del campo de la investigación educativa, hemos de señalar que existen gran variedad de artículos y libros que analizan o citan en algún momento el papel de las actitudes del profesorado, alumnado, padres, equipos directivos de centro, administración... Bell y otros (1988: p. 239) dicen que "la mayor parte de los profesores dan considerable importancia a la promoción de actitudes favorables en sus clases de matemáticas". Gómez Chacón, investigadora matemática en estos temas, dice en su artículo "Un instrumento para la autorregulación de las emociones en Matemáticas" (1997, p. 5): "En los últimos años distintos autores han concedido un papel importante a las estrategias que permiten al sujeto la toma de conciencia de la actividad mental, concediéndole gran relevancia a los aspectos cognitivos (metacognición). Sin embargo se ha prestado menor atención a la toma de conciencia de la actividad emocional". La introducción de ésta "permite hacer valer el derecho que tienen las emociones a jugar un papel significativo facilitador o debilitador del aprendizaje y a que la calidad emocional de las interacciones en clase ejerza una influencia significativa en lo que se aprende".

Por ello en el mismo artículo Gómez Chacón (1997, p. 5) habla de la alfabetización emocional, que "engloba habilidades tales como el control de los impulsos y fobias en relación con la asignatura, control que permite desarrollar la necesaria atención para que se logre el aprendizaje, la autoconciencia, la motivación, el entusiasmo, la perseverancia, la empatía, la agilidad mental, etc."

Uno de los grandes problemas que se plantean en la enseñanza de las Matemáticas (y de la enseñanza en general) queda reflejada en la rueda de Dyer: 


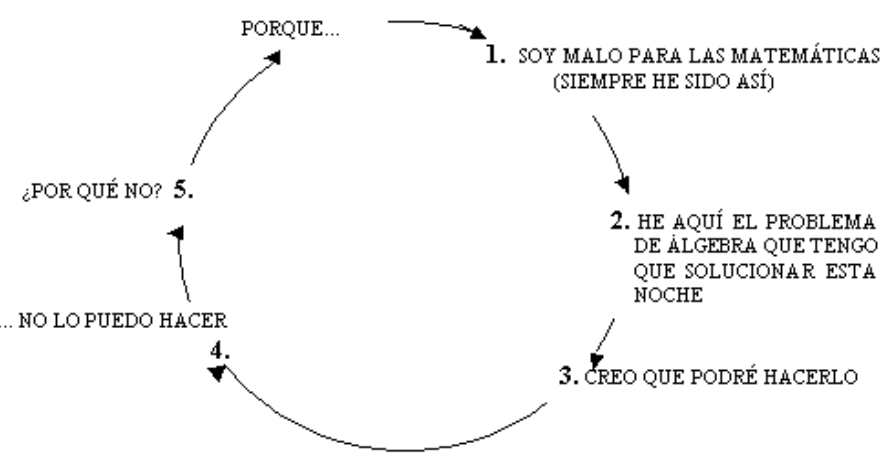

(AL CABO DE 10 MINUTOS)

En muchas ocasiones se acepta el paso del 3 al 4 como algo que viene impuesto, sin buscar soluciones ni luchar contra esa situación. "No tiene sentido aceptar esa actitud tan general de considerar las Matemáticas como disciplina tediosa, difícil de entender e inútil. Se trata de buscar soluciones constantemente...". Chamoso y Miguel (1995: p. 322). Y para eso necesitamos varias cosas en el marco que hemos diseñado. En primer lugar, una actitud positiva del alumno hacia la enseñanza. En segundo lugar, una preocupación del profesor (no se puede dejar de señalar la importancia y la necesidad de una actitud favorable del profesor). $Y$ en tercer lugar una apertura a cualquier tipo de recursos para la enseñanza de las Matemática. Esto último no es algo separado de los otros dos anteriores, sino más bien una ayuda para poder desarrollarlos. Vamos a centrarnos en él.

\section{Los recursos en la enseñanza de las Matemáticas.}

Los recursos deben ser un medio y no un fin para lo que pretendemos. Para ello tendremos que responder a la pregunta: ¿Qué buscamos? ¿Qué queremos hacer? ¿Qué intentamos conseguir? Es decir, cuando vamos a explicar un concepto, o trabajar con algo, tenemos que pensar siempre para qué lo queremos. Eso es fundamental cada vez que se hace cualquier cosa. Buscamos los recursos o materiales concretos más adecuados para explicar nuestro concepto en ese momento. Pero nunca podemos pretender diseñar una clase con la única idea de que sea atractiva y de que guste, o para entretener y llenar la última clase antes de vacaciones. Se debe perseguir el medio que nos permita llegar mejor a la idea de lo que queremos explicar. Se trata de implantar una forma de trabajo. El material, el juego, las nuevas tecnologías... pueden ayudar, pero no son en sí mismos la solución de nada. Son un instrumento y no un fin.

Pero hasta ahora, cuando tenemos que explicar un contenido, por ejemplo, la divisibilidad, lo tenemos fácil. Jugamos prácticamente sobre seguro: elegimos entre los libros y material que existen lo que encontremos sobre el tema, pensamos qué y cómo puede ser la mejor introducción, el mejor final, los ejercicios más adecuados, el mejor desarrollo... llevando cada parte a su sitio correspondiente, basándonos en nuestra formación y en la experiencia, y adecuándonos al nivel y circunstancias de nuestros alumnos. Sin embargo, cuando buscamos un recurso o un juego no tenemos una variedad donde elegir. No existe una tradición y una experiencia general sobre el tema. En la Facultad de Matemáticas ni siquiera nos hablaron de que existiese algo para el estudio o la enseñanza de las Matemáticas distinto de la pizarra o de papel y lápiz. Pero cada vez van surgiendo más cosas. Tardaremos en encontrarlas, e incluso quizás tendremos que inventarlas y prepararlas nosotros. Paciencia. La vida es dura.

En las Matemáticas actuales se atenúa el rigor excesivo. Se busca que la enseñanza sea significativa. La frase que reina es "aprender a aprender" y a aplicar las Matemáticas. Se trata de sustituir la clase magistral. No de forma vengativa pues tiene muchas cosas buenas, sino ante el cambio de los tiempos y de los puntos de vista. La memoria, por ejemplo, ha sido considerada tradicionalmente como uno de los pilares de la inteligencia humana. Y realmente quizás lo sea, pero no de forma aislada. Lo que llega a una persona se almacena en la memoria, pero en muchas ocasiones permanece allí durante un espacio muy corto de tiempo, especialmente si la información no es significativa en sí misma o no se encuentran formas de relacionarla con conocimientos previos. Hemos de tener en cuenta que un buen conocimiento matemático se debe basar en los conocimientos anteriores, es decir, en la memoria, pero siempre que ésta sea significativa.

Se quieren introducir nuevas formas de enseñanza, sobre todo métodos de enseñanza constructivistas, enseñanza por descubrimiento o expositivo significativo, para que cada uno vaya construyendo su propio conocimiento. Se quieren hacer unas Matemáticas agradables y atractivas que superen los tradicionales miedos y angustias. Se intenta sustituir el trabajo individual por un trabajo más colectivo, buscando también en ese grupo una mayor cooperación. Miguel de Guzmán (1987: p. 13) decía que "a los contenidos de enseñanza matemática se les suele atribuir una importancia relativa que probablemente no merecen. La Matemática es mucho más una ciencia de método que de contenido". Y además de los recursos no podemos olvidar el trabajo en grupo. Con mucho cuidado, porque están en nuestras manos personas, seres humanos a los cuales podemos condicionar. Queremos escuchar sus ideas y discutirlas, explorar las ramas de sus sugerencias, participar con ellos en el desafío de sus argumentos y celebrar sus descubrimientos con ellos para repetir el ciclo de nuevo hasta llegar a otros niveles de pensamiento analítico. Hay que arriesgar. Tenemos que buscar, escribir, formalizar. Con trabajo y dedicación las cosas no pueden salir mal. Tenemos que ir dando pasos poco a poco, pero pasos firmes para que nosotros mismos u otros puedan recoger frutos de lo que hemos sembrado, con sus errores y sus aciertos.

No podemos olvidar nunca una norma muy importante: el sentido común. Como dice Mark Saul (1997: p. 184) en su artículo "Common Sense: The Most Important Standard", "Las Matemáticas tienen muchas facetas y son posibles muchos caminos de enseñanza. Encontramos en nuestras aulas todo tipo de estudiantes; podemos utilizar muchos estilos de enseñanza para conseguir llegar a ellos. Para todo ello tenemos que utilizar el sentido común". De ese modo podremos aplicar las reglas más generales a cada situación concreta.

Para conseguirlo tenemos que concienciar a nuestros alumnos de que todos perseguimos lo mismo, que la finalidad de todos es aprender de la mejor manera posible la mayor cantidad de cosas en el tiempo de que disponemos, en un "codo a codo" de los alumnos con el profesor, con el departamento y con la sociedad en general. Se tiene que acabar la idea de que el profesor es el "jefe" (todos hemos conocido casos de profesores que dejan de explicar una parte para luego preguntarlo en el examen; o que ante una cierta pregunta concreta de un estudiante diga: "eso no lo puedo contestar porque mañana cae en el examen") al cual se le tiene miedo porque es el que pone las notas, sino más bien un compañero, al que hay que respetar en grado máximo y nos va a ayudar siempre que pueda. 
Tenemos que intentar que cada hora de clase sea una obra de arte. Recordemos la anécdota de Dyer, cuando un profesor le dice a sus compañeros que llevan más de 30 años dando clase, "¿Han estado ustedes realmente enseñando 30 años, o han estado enseñando un año 30 veces?" ¿Por qué cuando se habla de creatividad generalmente se piensa en pintura, danza, música e incluso literatura, pero pocas veces se ven las Matemáticas como áreas creativas? ¿Por qué se las sigue concibiendo como una ciencia en que únicamente existe la repetición y la memoria? Las Matemáticas son eminentemente creativas, y eso es lo que tenemos que demostrar en las escuelas. Tenemos que alejar esa idea popular de que el aprendizaje, para que sea bueno, tiene que ser desagradable.

¿Es esto una nueva matemática? Es la Matemática de siempre. Pero vista de otra forma, más cercana y más dentro de la vida diaria.

Pero, ¿por qué ponemos ese énfasis en la importancia de los recursos? Enseñar es responsabilidad del profesor y de toda la sociedad, así como aprender lo es del propio alumno. Y queremos que cada uno vaya construyendo su propio conocimiento. Para que esto sea lo mejor posible tenemos que buscar todos los recursos existentes. En los años tempranos de la edad escolar, nuestros alumnos han vivido multitud de experiencias para dominar la capacidad de comunicar, manipular, interpretar lo que ven y adaptarlo a las circunstancias. Algo común de esas experiencias es la capacidad de los alumnos de transformarlas de una expresión a otra. La idea de la transformación como un recurso puede ser entendida como una herramienta (Association of Teachers of Mathematics, 1985: p. ix-xvi). Hacer una transformación significa una experimentación dinámica de la articulación de una situación matemática. Se trata de que llegue a ser familiar la interconexión entre los pensamientos y la estructura del recurso que sea. Sencillamente, muchas veces lo que pasa con ellos es que buscan "mantener el valor pero cambiar la apariencia". Esto es lo que ocurre, por ejemplo, con algo escrito en un papel, que es la representación del pensamiento, o la representación de los bloques de Dienes para demostrar la estructura de número.

Realmente reconocemos que existen recursos válidos porque los hemos conocido desde nuestra juventud, porque los hemos usado de diversas formas durante los años que hemos estado aprendiendo. Pero se busca que, para llegar a la mente de los estudiantes, todos estos caminos dependan lo menos posible del azar y de las circunstancias del momento de utilizarlos. Es decir, que tengan una buena preparación previa, seria y rigurosa. Eso sucede, por ejemplo, en el deporte. En general un atleta, cuando ha realizado una planificación y preparación buena, seria y rigurosa, los resultados no suelen depender de las circunstancias o del azar (aunque no se consiga exactamente el primer puesto). Sin embargo eso no ocurre tanto en un equipo de fútbol, que depende mucho más del azar.

¿Cómo se pueden organizar todos estos recursos para la enseñanza? De muchas maneras y con muchos criterios, pero desde nuestro punto de vista parece más importante subrayar que, para poder utilizar cualquiera de ellos, tenemos que seguir unos pasos:

1. Reconocerlo, tener conciencia de que existe.

2. Admitir que se necesita, creer en él.

3. Saber utilizarlo.

\section{$1^{\circ}$ Reconocerlo, tener conciencia de que existe}

Es tarea difícil poder utilizar y aplicar recursos en la enseñanza si realmente los docentes no los conocen. En una ocasión, hace años, hablando con un grupo de 40 recién licenciados en Matemáticas sobre las directrices más importantes del nuevo Sistema Educativo, discutíamos sobre la necesidad de introducir en el aula materiales y otros recursos para motivar a los estudiantes en una disciplina tan importante como las Matemáticas. La actitud escéptica y la oposición férrea fue completamente general y enseguida surgió la frase: "las Matemáticas son algo demasiado importante como para estudiarlas con materiales y jueguecitos". O: "Eso las hará más receptivas y amenas, pero ni mucho menos ayudará a entenderlas mejor". O bien: "Las Matemáticas tienen que ser difíciles. Hay que sufrir. En caso contrario no son Matemáticas".

Traté de explicarles que cierto material, como por ejemplo los bloques lógicos, puede acercarnos de forma muy sencilla a algo tan importante como puede ser entender qué es un teorema y qué es una definición. Además, ese mismo material se podía utilizar de forma positiva desde niños de tres años hasta personas de cualquier edad, pudiendo plantear problemas lógicos que el adulto más preparado no encontraría sencillos.

Ante mi insistencia, por fin alguien preguntó: "Pero, ¿qué es eso de los bloques lógicos?". Quedé perplejo al darme cuenta de que ni uno solo sabía lo que eran, y ni siquiera los habían visto nunca. Y aún más: me di cuenta de que casi la totalidad no conocía prácticamente ningún material para utilizar en clase de Matemáticas distinto de la pizarra (sin duda muy importante, pero quizás insuficiente). Realmente, por no conocer los bloques lógicos uno no va a ser peor docente, ni peor matemático ni peor ciudadano pero, si no se conocen, ¿cómo se puede decir si son buenos o malos? Quizá ésta sea la primera causa de la dificultad de mentalizar a los docentes para la utilización de materiales en un aula de Matemáticas.

El ordenador también nos da una buena prueba de ello. Es de sobra conocido lo que es, pero muy pocos conocen sus posibilidades. Incluso actualmente muchas de ellas están todavía por descubrir o lo van haciendo poco a poco, como los programas interactivos y multimedias, y la rapidez y las posibilidades de conexión de Internet a distancia al instante.

Hoy día es relativamente fácil conseguir una larga lista de recursos disponibles para la enseñanza de las Matemáticas. Basta con recurrir a diversos catálogos. No obstante, esos recursos son inútiles si nuestra reacción no es más que saber que existen, conocer sus nombres y muchas veces ni eso, como en el ejemplo anterior. Para ello es muy importante la actitud del profesor ante la enseñanza.

\section{$2^{\circ}$. Admitir que se necesita, creer en él}

Realmente, si no se conocen no se pueden aplicar. Pero aunque se conozcan es necesario un paso intermedio para su uso en el aula, que es creer en esos recursos, pensar que son adecuados, que tienen posibilidades para lo que queremos explicar. Y que no cumpla únicamente el requisito de ser distinto con el fin de amenizar la clase, o que sirva de diversión de los estudiantes. Esa no puede ser nuestra pretensión. Tiene que cumplir el fin propuesto según nuestro criterio.

Muchos opinan que el ordenador es un recurso importante en la enseñanza de la Matemática. Otros que también lo son la calculadora, el ábaco, los cubos encajables, las regletas, y muchos otros que "permiten materializar y manipular aspectos matemáticos que, sin esta ayuda, 
podrían resultar excesivamente abstractos" (Primaria, M.E.C., 1992: p. 123). Pero, ¿qué piensa un docente en su propia aula? En los textos de Matemáticas aparecen términos como examinar, representar, resolver, mostrar, probar... ¿Cómo puede realizar su trabajo? Depende de su actitud y orientación pedagógica.

Es casi seguro que muchos de nosotros admitimos que existen los recursos. Lo admitimos como adultos, y adultos con experiencia en Matemáticas. Entendemos el valor posicional, utilizamos fácilmente los algoritmos preferidos, tenemos bien formadas nuestras estrategias de cálculo mental... De esta forma podemos "ver" la Matemática, y apreciar que existen aspectos matemáticos detrás de cada recurso. Esa es la conexión que nosotros vemos. Hemos puesto nuestro pensamiento propio dentro de ellos. Con nuestra formación es fácil ver las posibilidades que presentan para demostrar una idea abstracta. Estamos sumergidos en la construcción de nuestro propio entendimiento y podemos ver claramente esos conceptos en recursos escogidos para la clase de Matemáticas.

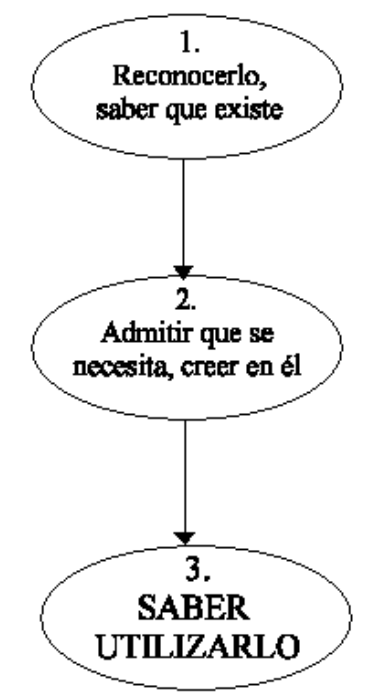

\section{$3^{\circ}$. Saber utilizarlo}

En letras mayúsculas. Una vez que se conocen los recursos, y que se admite que son buenos para lo que se pretende, hay que utilizarlos en la forma adecuada, dentro del contexto de contenidos concretos, teniendo en cuenta el grupo de alumnos a los que van dirigidos, el momento en que se van a utilizar... Tienen que servir de forma selectiva para poder comunicar la Matemática, para crear un ambiente que ayude a hacerlo, para que los estudiantes participen en el intercambio de ideas, para que describan lo que hacen, ven y piensan, para que les permitan probar sus hipótesis... Este es el punto más importante porque es el de actuación directa, pero no tiene sentido si antes no se ha atravesado por los pasos 1 y 2 .

En algunos momentos los docentes nos saltamos el paso 2, pasando de conocer algo a utilizarlo sin admitir que se necesita, sin tener siquiera confianza de que lo que usamos sea bueno y válido para lo que pretendemos enseñar. $\mathrm{O}$ incluso en ocasiones llega a nuestras manos, por ejemplo un CD-ROM, y sin estudiarlo nos hemos puesto a utilizarlo en clase con intención de hacer algo distinto, es decir, empezando directamente por el punto 3. Indudablemente, los resultados en general no pueden ser buenos. Si el profesor no se muestra convencido de lo que hace como docente, conociendo con detalle el recurso utilizado y sus posibilidades educativas, es difícil que lo pueda llevar al aula de forma adecuada ni pueda transmitir entusiasmo.

Se busca dar a los estudiantes un contexto matemático. Pero, cuidado, esto no quiere decir que los recursos lleguen a tener preponderancia sobre la lección, sino que el docente organiza ésta alrededor de ellos. Recordemos que no se tiene que ser esclavo de los recursos. Según la construcción del maestro o profesor, el contexto ofrece muchas posibilidades de inmersión en la Matemática, Durante la interacción con los alumnos, posiblemente una variedad de construcciones matemáticas afloran en la clase. El docente tiene que estar preparado para todas ellas. Con una mente abierta tiene que permitir que los estudiantes expliquen y demuestren lo que van construyendo respecto a algún aspecto de la Matemática. Y es importante la labor del maestro o profesor de penetrar seriamente en el razonamiento de los alumnos. Los recursos sirven para ayudar en este proceso y clarificar esta línea de pensamiento.

De la multitud de resultados del estudio TIMSS (1997), es significativo lo que apunta el director en su interpretación particular de éstos: el libro de texto es la fuente principal que la mayoría de los profesores utilizan para decidir cómo presentar un tema en su clase. Por eso, ¿hay un espacio para los recursos?

\section{BIBLIOGRAFÍA}

AL CUOCO (1995): Some Worries About Mathematics Education. Rev. The Mathematics Teacher, Vol. 88, $\mathrm{n}^{\circ}$ 3, March, p. 186-187. ALCALÁ, M. (1996): Enseñanza de la Matemática y niveles operatorios. Actas $8^{\circ}$ J.A.E.M., Salamanca, p. 51-56.

ALPER, L.; FENDEL, D.; FRASER, S. y RESEK, D. (1995): Is this a Mathematics Class?. Rev. The Mathematics Teacher, Vol. 88, ${ }^{\circ} 3$, March, p. 186-187.

ALSINA, C. (1996): Enseñar matemáticas. Ed. Grao. Barcelona.

ASSOCIATION OF TEACHERS OF MATHEMATICS (1985): Notes on Mathematics for Children. ATM. Derby, p. ix-xvi.

BELL, A.W.; COSTELLO, J. y KÜCHEMAN, D. (1988): Research on Learning and Teaching. Ed. Nfer-Nelson. Oxford, p. 239-257.

BUJANDA JÁUREGUI, Ma.P. (1996): La atención a la diversidad en la Educación Obligatoria. Actas del IV Congreso Regional Castellano-Leonés de Educación Matemática. Valladolid, p. 9-21.

CHAMOSO SÁNCHEZ, J. Ma . (1996): Materiales y recursos de Matemáticas: aplicación en el aula. Actas del IV Congreso Regional Castellano-Leonés de Educación Matemática. Valladolid, p. 71-73. 
CHAMOSO, J. Ma y MIGUEL, E. (1995): Materiales y recursos didácticos para la enseñanza de las Matemáticas. Rev. de Enseñanza e Investigación educativa AULA. Ediciones Universidad de Salamanca. Salamanca, p. 317-329.

CUESTA DUTARI, N. (1981): La Sinfonía del Infinito. Ediciones Universidad de Salamanca. Salamanca, p. 4.

DEPARTMENT FOR EDUCATION, DfE (1995): Mathematics in the National Curriculum. HMSO. London.

DEPARTMENT OF EDUCATION AND SCIENCE, DES (1982): Mathematics Count. HMSO. London.

DIENES, Z.P. (1970): La construcción de las matemáticas. Vicens-Vives. Barcelona, p. 5.

DYER, W. (1992): Tus zonas erróneas. Ediciones Grijalbo. Barcelona.

GÓMEZ CHACÓN, I.Ma . (1997): Una instrumento para la autorregulación de las emociones en Matemáticas. Boletín I.E.P.S. $n^{\circ}$ 71, diciembre, p. 5-7.

GRUPO DECA (1990): Didáctica de la resolución de problemas. CEP de Burgos. Burgos, p. 144-145.

GUERRERO OJEDA, J. (1989): Ámbitos y funciones del curriculum matemático. Epsilon, Rev. de la S.A.E.M. Thales no 14, p. 57-62.

GUICHARD, J.P. (1986): Didactique des Mathématiques: le dire et le faire. Ed. Cedic-Nathan. París.

GUZMÁN, M. de (1987): Cuestiones fundamentales de la enseñanza de la Matemática. Rev. Thales n1 8, p. 13-26, 1987.

I.E.P.S. (1997): Nuevo concepto en la enseñanza: la alfabetización emocional. Boletín I.E.P.S. $n^{\circ} 71$, diciembre.

I.N.C.E. (1995): Datos básicos sobre la Evaluación de la Educación Primaria en España. M.E.C.

MALÉN AZNÁREZ (1977): Ni ogro, ni aburridas. El País Semanal, mayo.

M.E.C. (1992): Primaria. Área de Matemáticas. Secretaría de Estado de Educación. Madrid, p. 123.

SAUL, M. (1997): Common Sense: The Most Important Standard. Rev. The Mathematics Teacher,Vol. 90, $n^{\circ}$ 3, March, p. 182-184.

SANTALÓ, L.A. (1997): Matemáticas para profesores. Rev. Epsilon no 38, p. 199-208.

TIMSS (1997): Tercer Estudio Internacional de Matemáticas y Ciencias.

TIMSS (1997): Tercer Estudio Internacional de Matemáticas y Ciencias. Nfer, Slough.

(C) Ediciones Universidad de Salamanca.

Páginas mantenidas por Francisco Ignacio Revuelta Domínguez

y Luis González Rodero

webmrte@usal.es 